\title{
A rare case of chronic benign tracheo-oesophageal fistula, with Candida albicans cultured from a pleural effusion
}

\author{
${ }^{1} \mathrm{P}$ Stride, ${ }^{2} \mathrm{M}$ Stare, ${ }^{2} \mathrm{~L}$ Kelly, ${ }^{3} \mathrm{R}$ Horvath, ${ }^{4} \mathrm{~T}$ Wood, ${ }^{5} \mathrm{C}$ Alexander \\ ${ }^{1}$ Academic Head; ${ }^{2}$ Medical Registrars; ${ }^{4}$ Consultant Radiologist; ${ }^{5}$ GMC3 Student, University of Queensland School of Medicine, Redcliffe \\ Hospital, Queensland, Australia; ${ }^{3}$ Infectious Diseases Physician, Prince Charles Hospital, Brisbane, Queensland
}

ABSTRACT We present a case of chronic benign tracheo-oesophageal fistula in an immunologically competent elderly female, cured with a period of nasogastric feeding.

KEYWORDS Benign chronic tracheo-oesophageal fistula, pleural effusion, Candida albicans

DECLARATION OF INTERESTS Dr Stride has received payment as a speaker for pharmaceutical companies Amgen, Sanofi and MSD.

\author{
Correspondence to P Stride \\ University of Queensland School \\ of Medicine, Redcliffe Hospital, \\ Redcliffe, Queensland, Australia
}

tel. 0610732567980

e-mail pjostride@gmail.com
Tracheo-oesophageal fistulas are most frequently found in paediatrics as a congenital abnormality. In adults it is usually an acute problem associated with malignant disease or trauma which may be external or postprocedural. We describe a chronic case in an elderly patient in the absence of trauma or malignant disease.

\section{CASE REPORT}

An 88-year-old woman was admitted with a two-week history of a productive cough expectorating yellow sputum. She had also experienced swallowing difficulties for a year, with coughing episodes after ingesting solids and liquids. Past history included atrial fibrillation, cervical spondylosis, asthma, Parkinson's disease and hypothyroidism. On admission, the patient was on the following medication: thyroxine $100 \mu$, digoxin $125 \mu$, levodopa $250 \mathrm{mg}$ plus carbidopa $25 \mathrm{mg}$ (sinemet 25/250) three times a day, celecoxib $200 \mathrm{mg}$ as needed and salbutamol metered aerosol $100 \mu \mathrm{g}$ also as required. She had a history of a prolapse repair, hysterectomy and dental surgery, but her last general anaesthetic with intubation was at least thirty years previously. She said that she had never been treated for osteoporosis or taken oral bisphosphonates. On examination the patient appeared cachectic but was mentally alert. Abnormal observations included her weight of $35 \mathrm{~kg}$, her body mass index (BMI) of 14 , a heart rate of 88 beats per minute in atrial fibrillation and a temperature of $37.2^{\circ} \mathrm{C}$. Chest auscultation detected bilateral basal crepitations. Straw coloured fluid $(640 \mathrm{ml})$ was aspirated from a left basal pleural effusion.

\section{INVESTIGATIONS}

Initial pathology results were as follows: haemoglobin $107 \mathrm{~g} / \mathrm{L}$, white blood cell count $5,400 / \mu \mathrm{L}$. The only abnormalities in a standard biochemical profile were urea $10.3 \mathrm{mmol} / \mathrm{L}$ (reference range [RR] 2.9-8.2), and albumin $30 \mathrm{~g} / \mathrm{L}$ (RR35-50). C-reactive protein was raised at $77 \mathrm{mg} / \mathrm{L}$.

A chest $X$-ray (Figures IA and B) revealed a moderate sized left-sided pleural effusion, with left basal atelectasis. The film also showed some right upper zone pleural scarring, diffuse increased interstitial markings, vascular plethora and cardiomegaly suggestive of superimposed cardiac failure. Computerised tomography (Figures 2A and $B$ ) of the chest revealed a fistula linking the trachea and the oesophagus.

Analysis of pleural aspirate revealed the following abnormalities: protein $14 \mathrm{~g} / \mathrm{L}$, glucose $5.4 \mathrm{~g} / \mathrm{L}$, LDH 100 $\mathrm{IU} / \mathrm{L}$, albumin $<5 \mathrm{~g} / \mathrm{L}$. Microscopy detected scant leucocytes and gram positive cocci, yeast $\mathrm{I}+$, unsuitable for cell count. Culture: Escherichia coli 2+, Candida albicans 2+, Enterococcus 2+, Staphylococcus aureus 2+, Pseudomonas sp 2+ and Klebsiella pneumoniae 2+. Upper gastroesophageal endoscopy revealed a benign-appearing small fistula $22 \mathrm{~cm}$ from the mouth, with no evidence of malignant disease.

\section{TREATMENT}

Following the identification of a tracheo-oesophageal fistula (TOF), the patient was treated with fluconazole 200 $\mathrm{mg}$ daily plus piperacillin $4 \mathrm{gm}$, tazobactam $0.5 \mathrm{gm}$ three times a day and underwent re-feeding with a nasogastric tube. Blood biochemistry was monitored daily, with a focus on the glucose, phosphate, potassium and magnesium measurements. Appropriate replacement therapy to prevent re-feeding syndrome was also provided.

Following discussions with thoracic, surgical and gastroenterology teams, it was decided to treat her with an oesophageal stent rather than surgery due to her age and frailty, and observe progress. After three weeks of 

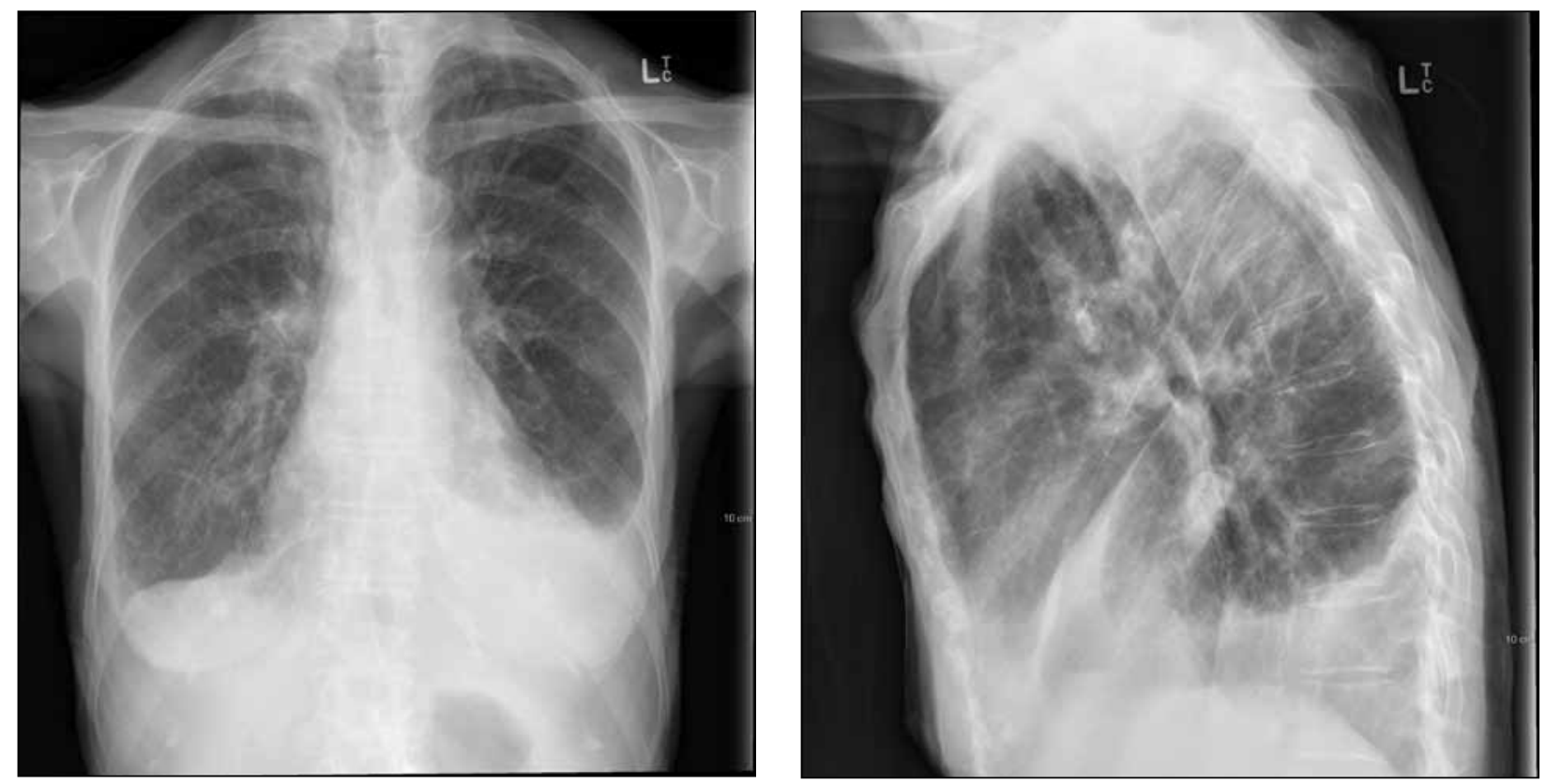

FIGURES IA AND B Posterior/anterior and lateral chest X-rays showing a pleural effusion.

oesophageal bypass with nasogastric feeding, repeat oesophagoscopy and barium swallow showed no evidence of the TOF. Resolution of the fistula with nasogatric tube bypass was successful and normal feeding was recommenced without problem.

\section{DISCUSSION}

Our patient had the rare problem of a chronic idiopathic benign TOF with Candida albicans cultured from a pleural effusion. Uncontrollable coughing after fluid ingestion is characteristic of a TOF (also known as Ono's sign). Candida infection of the mucosal surfaces is an unpleasant

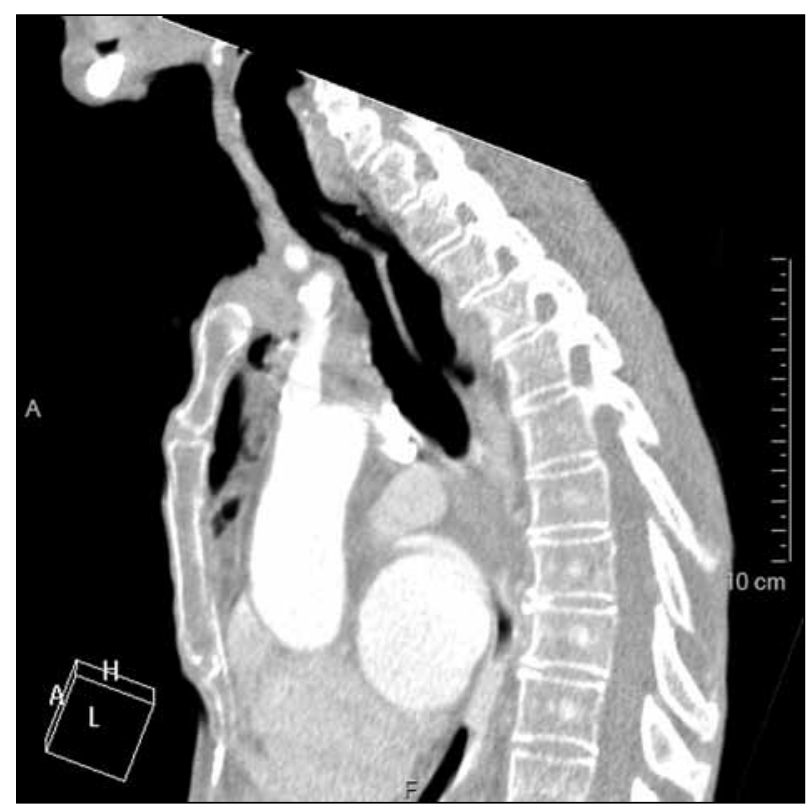

though rarely serious condition. Systemic Candida infection in the blood or internal body fluids is a much more serious problem often associated with severe disease or an underlying cause. Labelle' and Shorr ${ }^{2}$ reviewed separate series of 738 and 245 patients with Candida bloodstream infection and found mortality rates of $29.4 \%$ and $28.3 \%$, respectively. Candida infection in addition to all the other microorganisms grown in our case strongly suggested an intestinal fistula.

Diddee $^{3}$ reviewed the problem of acquired TOF in adults and found that $50 \%$ are due to malignant disease, and nearly all the remainder are due either to external trauma, internal trauma (usually iatrogenic such as

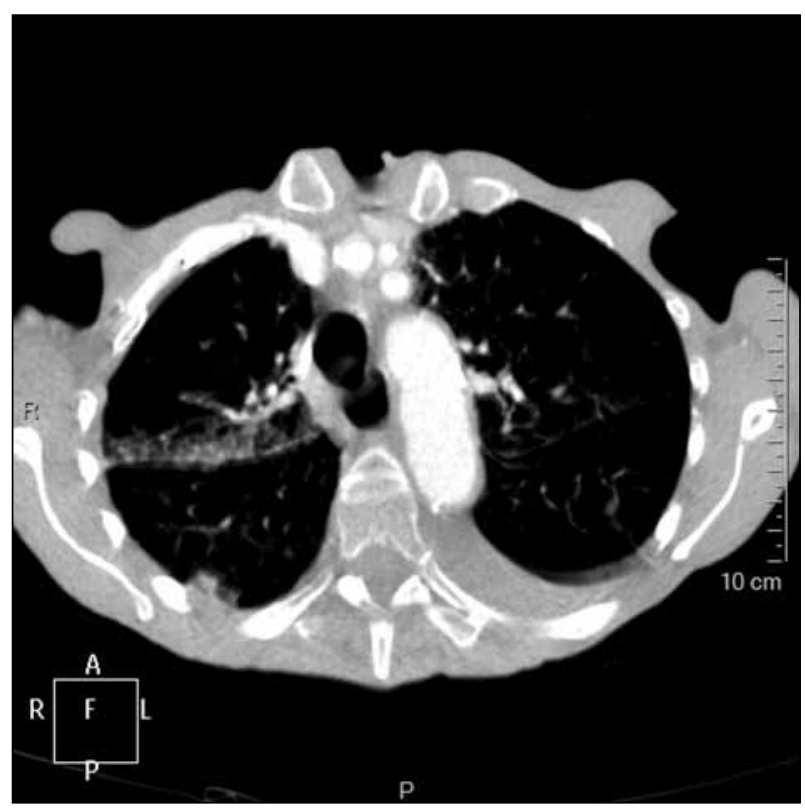

FIGURES 2A AND B Computed tomography scans of the thorax, showing a tracheo-oesophageal fistula. 
surgery), intubation and endoscopy, or due to the ingestion of corrosive fluids or button-type batteries. Surgical closure if possible is recommended, but extremely careful isolation and protection of the airway is required.

Darbari ${ }^{4}$ described seven patients with a non-malignant TOF. One was a child with a delayed presentation of a congenital malformation and six were due to trauma, three from ingested objects or substances, one to external trauma and two following severe vomiting.

Alkrinawi ${ }^{5}$ described 105 cases of children with pleural effusions, only one of whom had Candida cultured from the pleural aspirate. $\mathrm{Ko}^{6}$ reviewed 67 cases of fungal empyema detected over an eight-year period in Taiwan. Of the 73 fungal isolates in these patients, 47 (64\%) were Candida species ( 28 with $C$ albicans, 13 with $C$ tropicalis, six with other species); $90 \%$ of these patients had underlying disease, $84 \%$ acquired the fungal isolates in the intensive care unit, $79 \%$ had impaired immunity, particularly those with cancer, diabetes, cirrhosis and on prolonged steroid therapy; and $60 \%$ were on a course of broad spectrum antibiotics. Overall mortality was $73 \%$. Fungal pulmonary infection is more commonly a parenchymal problem; $\mathrm{Chen}^{7}$ found fungal isolates in a pleural effusion in sixteen of 140 patients from the same group in Taiwan with pulmonary fungal infection.

\section{CONCLUSION}

In summary, the occurrence of a non-malignant chronic idiopathic TOF present for perhaps a year in an elderly but otherwise immunologically intact patient, with an associated Candida albicans infected pleural effusion and cured with tracheal isolation, oesophageal bypass and nasogastric tube feeding, is a most rare occurrence. The detection of Candida albicans and a mixed, predominantly gram negative flora in the chest should alert physicians to the possibility of an enteric fistula.

\section{Postscript}

Four months after the original admission the patient was readmitted with pneumonia. A repeat computerised tomogram of the chest revealed that the fistula had reopened. The patient, an independent, frail woman, in full possession of her mental faculties and not depressed, refused treatment with a nasogastric tube or a percutaneous endoscopic gastrostomy feeding tube. She deteriorated, was transferred to palliative care and died. An autopsy was not performed. The outcome was finally unsuccessful and suggests that a considerably longer period of oesophageal bypass is the preferred treatment.

\section{REFERENCES}

I Labelle AJ, Micek ST, Roubinian N et al. Treatment-related risk factors for hospital mortality in Candida bloodstream infections. Crit Care Med 2008; 36:2967-72. http://dx.doi.org//0.1097/ CCM.0b013e31818b3477

2 Shorr AF, Gupta V, Sun X et al. Burden of early-onset candidemia: analysis of culture-positive bloodstream infections from a large US database.Crit Care Med 2009; 37:25 I9-26. http://dx.doi.org/ I0.1097/ CCM.0b0I3e3I8Ia0f95d

3 Diddee R, Shaw I. Acquired tracheo-oesophageal fistula in adults. Contin Educ Anaesth, Crit Care Pain 2006; 6:105-8. http://dx.doi. org/l0.1093/bjaceaccp/mkl019

4 Darbari A, Suryavanshi A, Tandon S et al. Non malignant tracheooesophageal fistula: our experience. Ind J Thorac Cardiovasc Surg 2005; 2 I: 272-6. http://dx.doi.org/ 10.1007/s I 2055-005-0005-7

5 Alkrinawi S, Chernick V. Pleural fluid in hospitalised paediatric patients. Clin Paediatr (Phila) 1996; 35:5-9. http://dx.doi. org/I0.II77/000992289603500I02

6 Ko SC, Chen KY, Hsueh PR et al. Fungal empyema thoracis: an emerging clinical entity. Chest 2000; II7;1672-8. http://dx.doi. org/10.1378/chest.II7.6.1672

7 Chen KY, Ko SC, Hsueh PR et al. Pulmonary fungal infection: emphasis on microbiological spectra, patient outcome, and prognostic factors. Chest 200I; 120:177-84. http://dx.doi.org//0.1378/chest. I20.I.I77 